\title{
Research Article Effect of TurboSwirl on Inclusions during Ingot Casting of Steels
}

\author{
Zhe Tan, Mikael Ersson, and Pär G. Jönsson \\ Division of Applied Process Metallurgy, KTH Royal Institute of Technology, Brinellvägen 23, 10044 Stockholm, Sweden \\ Correspondence should be addressed to Mikael Ersson; bergsman@kth.se
}

Received 2 April 2015; Accepted 14 July 2015

Academic Editor: Huaguang Zhang

Copyright (C) 2015 Zhe Tan et al. This is an open access article distributed under the Creative Commons Attribution License, which permits unrestricted use, distribution, and reproduction in any medium, provided the original work is properly cited.

\begin{abstract}
The use of TurboSwirl to obtain an improved steel cleanliness during filling of an ingot was numerically studied by VOF and DPM models. It was found that a radius-reduced TurboSwirl or a proper tapered mold entrance nozzle with an adequate developed region for steel flow can reduce the risk of mold flux entrapment in a mold. The ingot casting process can create highly turbulent conditions inside the mold during the initial stages of casting. Since the TurboSwirl generates much calmer filling conditions it can promote separation of large nonmetallic inclusions. The TurboSwirl also collects large inclusions $(200 \mu \mathrm{m})$ towards the axis of rotation, which should promote agglomeration. In addition, the residence time for inclusions of small sizes can be prolonged, increasing chance of agglomeration, which indirectly promotes their separation from steel. Moreover, the average turbulent dissipation rate in an ingot casting swirl setup is about $40 \%$ higher than that in a no swirl setup. This further facilitates the agglomeration of inclusions before they enter the mold. The removal of nonmetallic inclusions is thus enhanced because of an increasing inclusion collision rate due to both Stokes collisions and turbulent collisions, while maintaining a calm flow inside the mold.
\end{abstract}

\section{Introduction}

Nowadays, it is well accepted that steel cleanliness is of great importance in the steel industry to meet ever-increasing stringent demands on improved steel properties. Here, ingot casting is often used for production of specialty steel, where the quality is of first concern. More specifically, steel cleanliness becomes even more critical as it directly affects the yield and quality of the final product. Therefore, the control of nonmetallic inclusions during the uphill teeming process is of great interest to study from both theoretical and practical perspectives.

Based on their origins, nonmetallic inclusions which are present in the uphill teeming process can be classified into endogenous inclusions and exogenous inclusions. Endogenous inclusions represent mainly products from the secondary refining process. The size range of inclusions before vacuum degassing can reach values of up to $60 \mu \mathrm{m}$ and be smaller than $10 \mu \mathrm{m}$ after vacuum treatment [1]. Due to the fact that aluminum and silicon are the common reducing agents during the deoxidation process, alumina and silica make up the majority of the endogenous inclusions. Their morphological shapes may vary with the thermodynamic and kinetic conditions. Endogenous inclusions may also be generated during the precipitation during solidification. The fraction of this type of endogenous inclusions is usually small, but large clusters might form [2]. Exogenous inclusions are mainly formed as a result of reoxidation, mold flux entrapment, refractory erosion, and chemical reactions [2]. Exogenous inclusions usually have a relatively large size, which makes them detrimental to the quality of the final products. In addition, the source of exogenous inclusions comes from different locations [3]. During production, exogenous inclusions are generated from a combination of different sources, which make their composition complex. Hence, it is hard to fully eliminate the generation of exogenous inclusions during the steelmaking and teeming processes.

In order to minimize the inclusion content, it is necessary to study the growth and removal of nonmetallic inclusions. Many researchers have proposed several mechanisms which are considered to have an influence on inclusions growth $[2$, 4]. The growth of inclusions is controlled by diffusion of oxygen and deoxidant, Ostwald ripening, local thermodynamics, and Brownian motion when their size is small, that is, smaller than $1 \mu \mathrm{m}$. In collision due to velocity gradient in laminar shear zones, turbulent collisions and Stokes collisions are also 
found to be related to the growth of inclusions. Moreover, the significance of turbulent collisions and Stokes collisions has also been suggested [4-6]. After growing large enough, the inclusions can easily be removed to the mold flux layer by the steel flow due to their increasing buoyancy and by bubble floatation. Also, inclusions can be removed to the refractory surface. Overall, it is necessary to find a common solution to promote nonmetallic inclusions growth and removal so as to further improve the mechanical properties of steel products.

It has long been recognized that the flow pattern plays an important role during the uphill teeming process. An exogenous inclusions generation in the gating system and a mold flux entrapment in the mold have been found to be closely related to the flow pattern in the uphill teeming process [3]. Large macro inclusions generated by the steel flow in the horizontal runner have also been found in the steel sample from the horizontal runner [7]. In addition, the flow-induced wall shear stress can also result in an erosion of a refractory surface [8]. It is possible to modify the feeding system design to optimize the filling and solidification of ingots [9]. By introducing a flow pattern control device, the possibility of exogenous inclusions generation due to a flowinduced wall shear stress and a mold flux entrapment can be reduced [10]. In a recent study by the authors, a refractory geometry modification based on swirling flow generation component, TurboSwirl, showed a great potential to further reduce the amount of exogenous nonmetallic inclusions. The design of the TurboSwirl dates back to a 1914 patent [10], where at the time the numerical investigation of the system was impossible. The demand on steel cleanliness was also much lower compared today. In the precious paper by the authors, the number of exogenous inclusions generated at the initial filling was believed to decrease since the inlet flow conditions were much calmer compared to the conventional setup. Furthermore, the wall shear stress was also decreased due to the evenly distributed swirling flow [11]. There has also been recent work on producing swirl in continuous casting to improve the flow and temperature distribution in the continuous casting mold $[12,13]$. This illustrates that the applicability of swirling flow is not limited to ingot casting alone. In the present work, the potential of using TurboSwirl in uphill teeming to improve the steel cleanliness was further studied. The basic property of the TurboSwirl is to generate a swirling flow that can be used together with a flaring inlet into the mold to decrease the velocity of the steel as it enters the mold. The TurboSwirl setup can be seen in Figure 1.

\section{Numerical Modelling}

2.1. Inclusion Collision Theory. In steel flow two of the main collision mechanisms are turbulent collisions and Stokes collisions. The turbulent collisions model was originally developed to describe the collision of drops in turbulent clouds by Saffman and Turner [6]. In this model, the Kolmogorov length scale was used to find the minimum size of an eddy in a local homogeneous turbulent flow. Later the model was corrected and extended by other authors [5]. The model suggests that a well-designed gating system can

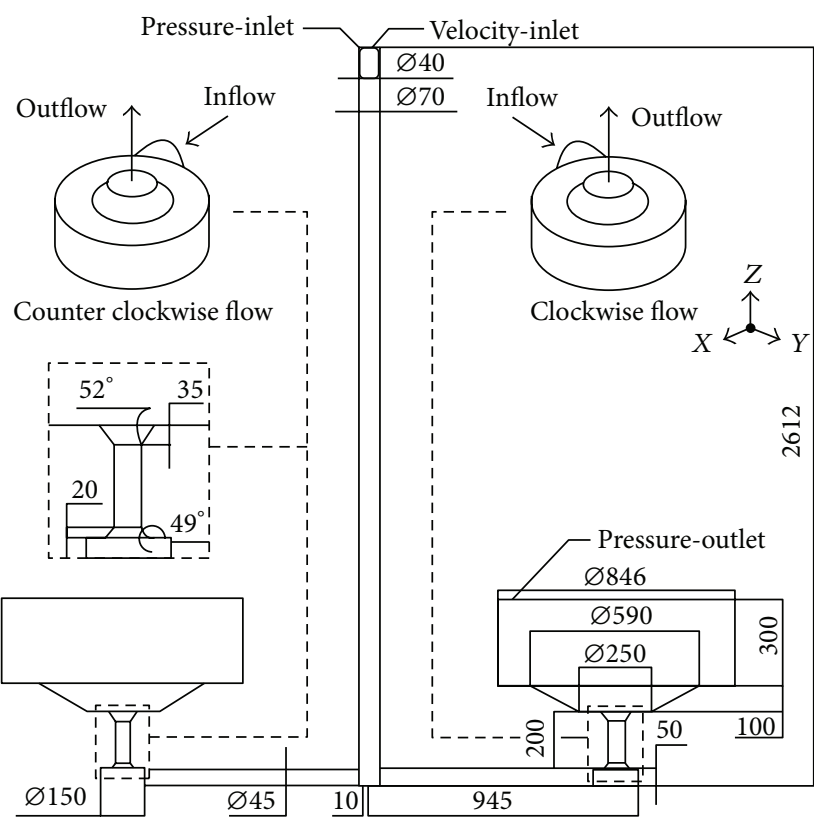

FIGURE 1: Schematic of the TurboSwirl setup for the uphill teeming process; note that the mold height has been reduced since the simulation only investigates the initial filling, units in $\mathrm{mm}$ [14].

promote turbulent collisions, $W_{t}$, by properly increasing the turbulence dissipation rate and the number concentration of inclusions in a relatively small volume, for example, bringing inclusions close to the axis of a rotational flow.

The size of the smallest eddies in a turbulent flow is given by the Kolmogorov length scale:

$$
\eta=\left(\frac{v^{3}}{\varepsilon}\right)^{0.25}
$$

where $v$ is the fluid kinematic viscosity and $\varepsilon$ is the turbulence dissipation rate. If the particle size is smaller than the Kolmogorov length scale, then the turbulence collision volume, $W_{t}$, is described by the following equations $[5,6,15]$ :

$$
\begin{aligned}
W_{t} & =1.30 \alpha\left(r_{i}+r_{j}\right)^{3}\left(\frac{\varepsilon}{v}\right)^{0.5}, \\
\alpha & =0.732\left(\frac{5}{N_{T}}\right)^{0.242} \text { for } N_{T} \geq 5, \\
N_{T} & =\frac{6 \pi \mu\left(r_{i}+r_{j}\right)^{3} \dot{\gamma}}{A}, \\
\dot{\gamma} & =\left(\frac{4 \varepsilon}{15 \pi v}\right)^{0.5},
\end{aligned}
$$

where $W_{t}$ is the turbulence volume collision rate, $r_{i}$ is the particle radius of particle $i, N_{T}$ is the ratio between viscous forces and Van der Waals forces, $\mu$ is the fluid viscosity, $\dot{\gamma}$ is the fluid deformation rate, and $A$ is the Hamaker constant.

When an inclusion is submerged in steel, it experiences a greater pressure from the bottom side than its top side. 
This pressure difference results in a net force upward, that is, buoyancy. Due to the nature of nonmetallic inclusions, they normally have a density which is less than that of steel. This causes the floatation of the inclusion in steel. During its floatation, a terminal velocity, $v_{t}$, is reached when the frictional force combined with the gravitational force exactly balances the buoyancy force. This terminal velocity is given by the following expression [16]:

$$
v_{t}=\frac{2}{9} \frac{\left(\rho_{p}-\rho_{f}\right)}{\mu} g r^{2},
$$

where $v_{t}$ is the terminal velocity, $\rho_{p}$ is the density of the inclusion, $\rho_{f}$ is the density of the steel, $\mu$ denotes the dynamic viscosity of steel, $g$ is the gravitational acceleration, and $r$ is the particle radius.

As can be seen from above, when two inclusions with different radii are subjected to floatation, the inclusion with a greater size will have a larger terminal velocity. This is quadratically proportional to the radius of the inclusion. Owing to this, the larger inclusion will catch up with the smaller inclusion. Hence, a larger difference in radius results in a faster collision.

When the reference frame is changed into a rotational field, the modified terminal velocity can be expressed as follows:

$$
v_{t}^{\prime}=\frac{2}{9} \frac{\left(\rho_{p}-\rho_{f}\right)}{\mu} a_{c} r^{2}
$$

where $a_{c}$ denotes the centripetal acceleration. In (7), the natural gravitational acceleration is not considered. The introduction of the modified terminal velocity depicts the tendency of inclusions with different sizes to gather towards the rotational center in a swirling flow. It can be found that an important factor which influences this tendency, besides the radius of an inclusion, is the centripetal acceleration. The concept of a modified terminal velocity will be discussed later in this study.

The Stokes volume collision frequency, $W_{s}$, has been suggested by previous researchers as shown below [5]:

$$
W_{s}=\frac{2 \pi \Delta \rho g}{9 \mu}\left(r_{i}+r_{j}\right)^{3}\left|r_{i}-r_{j}\right|,
$$

where $\Delta \rho$ is the difference in density between the particles and the molten steel, $r_{i}$ and $r_{j}$ are the particle radius of two different inclusions, and $\mu$ is the viscosity of the molten steel. Here, it can be found that the collision rate can drastically be reduced when inclusions have a similar size.

As shown above, the aim of promoting an improved collision volume is to separate nonmetallic inclusions from steel without them being entrapped inside the ingots after stripping. This can significantly improve the steel cleanliness. The collision volume of a collision mechanism can notably be decreased under certain circumstances. However, the collision rate for the system should be kept or increased in order to maintain the capability to separate nonmetallic inclusions from a steel bath. Therefore, the total collision volume, $W$, is of great concern and it can be written as follows:

$$
W=W_{t}+W_{s}+W_{\text {other }}
$$

where $W_{\text {other }}$ denotes the collision rate due to other collision mechanisms which may contribute.

2.2. Mathematical Model. Based on the results using a Volume of Fluid (VOF) model from the previous research by the authors [10], a Discrete Phase Model (DPM model) was adopted in the present work [17]. The inclusions injected were assumed to be alumina particles with a spherical shape and with a density of $3950 \mathrm{~kg} \cdot \mathrm{m}^{-3}$. Five different sizes, that is, 2 , $10,30,100$, and $200 \mu \mathrm{m}$, were adopted in the model. A oneway coupling was used and it was assumed that the fluid flow was not affected by the movement of the particles. This assumption was made considering that the volume fraction of inclusions is very low and the size of the inclusions is small, compared to the computational cells. Virtual mass force and pressure gradient force were used and a stochastic turbulence random walk model was used to include turbulence effects on the motion of the inclusions. Clustering, coalescence, and agglomeration were neglected. Moreover, the removal of inclusions to the refractory surface was not considered, mainly due to the lack of theory for deposition during swirling flows in high temperature melts. The turbulence was modeled by the realizable $k-\varepsilon$ model and care was taken to make sure that the size of the computational cells admitted $30 \leq y^{+} \leq 500$.

\section{Results and Discussion}

3.1. Improved Steel Cleanliness in the Mold. In order to produce an even swirling flow, the dimension of the TurboSwirl needs to be designed in such a way that the gas phase is separated from the liquid phase without causing an excessive entrapment of gas. This means that the height of the component needs to be large enough, without causing a too large steel loss at the end of casting due to the increased volume of the runner system. The swirl strength can be controlled by changing the radius of the TurboSwirl. A larger diameter decreases the swirl strength. Moreover, the mold entrance nozzle needs to be tapered so as to fit the development of the flow pattern in the vertical runner. Here, a full geometry model based on the previous studies $[3,11,14]$ was used to investigate the effect of the radius $(R)$ and mold entrance nozzle length $(H)$ variation on the hump height and on the difference between the hump height and the surface height $\left(H_{h}-H_{s}\right)$. The definitions of the important parameters are shown in Figure 2 and the results are shown in Figures 3 and 4 , respectively.

In Figure 3, the results show that a properly tapered vertical runner (mold entrance nozzle) with an adequately developed region for the steel flow can further reduce the initial hump height. There is an initial high hump height as the steel enters the mold (at around 2.5 to 3 seconds for the cases tested); then there is a slow increase in the hump height as the steel fills the mold. Observe the definition of the hump 


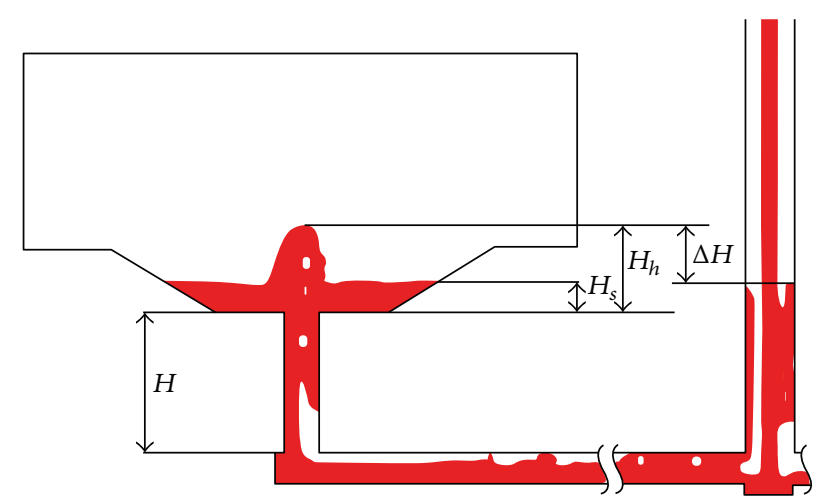

FIgURE 2: Definition of the hump height $\left(H_{h}\right)$, the surface height $\left(H_{s}\right)$, and the steel height difference $(\Delta H)$ [9]. The mold entrance nozzle height $(H)$ has been added in the figure.

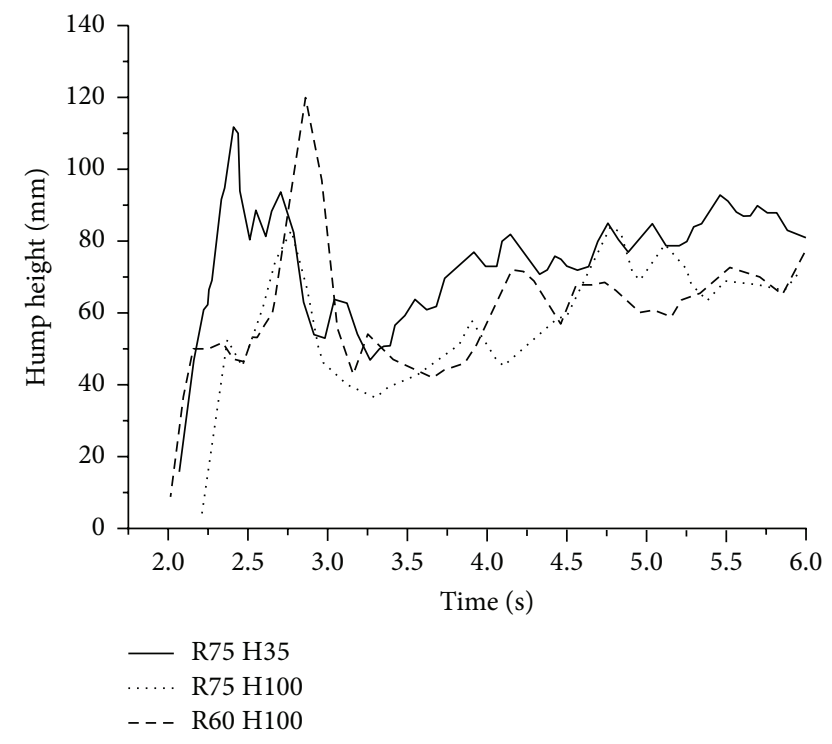

FIgURE 3: Effect of the TurboSwirl radius $(R)$ and mold entrance nozzle length $(H)$ variation on the hump height. The first steel appears in the mold at approximately 2 seconds from the start of the simulation.

height according to Figure 2. The steel surface also fluctuates slightly due to the transient nature of the filling and also due to the entrapped gas that originates from the trumpet region of the runner system. It is clear that a developing region of the swirl is beneficial when comparing the different mold entrance nozzle lengths (compare the $35 \mathrm{~mm}$ to the $100 \mathrm{~mm}$ ). There are however smaller benefits of generating a stronger swirl in this particular case as can be seen when comparing two different radiuses (75 and $60 \mathrm{~mm}$, resp.). It can also be seen that the smaller radius produces a somewhat higher initial splashing at approximately 3 seconds which is probably due to the decreased volume of the TurboSwirl. In conclusion it can be said that the TurboSwirl should be designed so as to minimize the volume of the TurboSwirl, while producing a calm inflow condition. A calmer initial inflow condition opens the possibility to lower the position of the mold powder

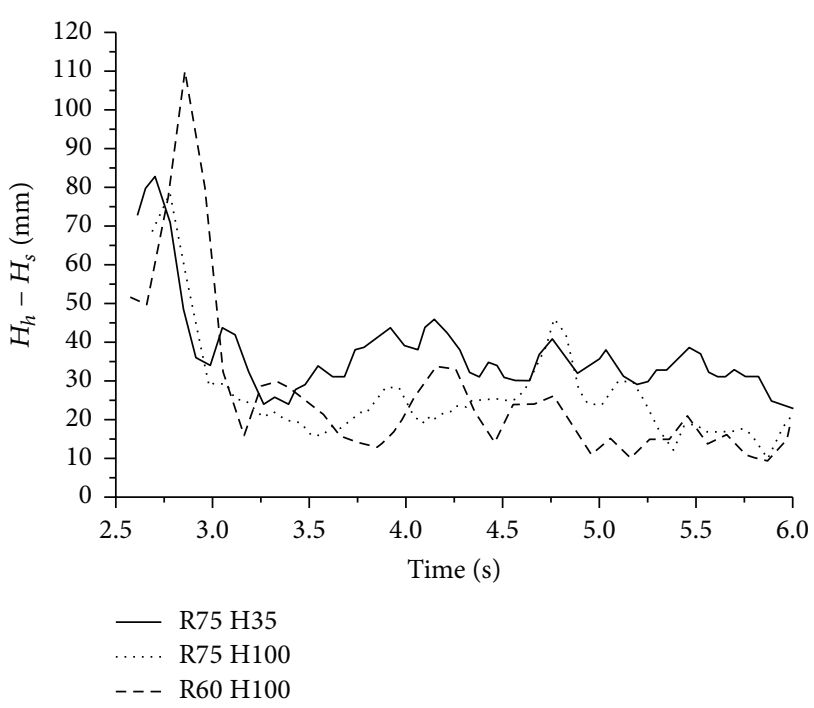

Figure 4: Effect of the TurboSwirl radius $(R)$ and mold entrance nozzle length $(H)$ variation on the difference between hump height and surface height (i.e., the visible hump on the free surface). The first steel appears in the mold at approximately 2 seconds from the start of the simulation.

bags which results in an earlier release of the mold powder and as such a smaller contact time for the steel flow with oxygen in the atmosphere.

In Figure 4, the visible hump height is investigated. It can be seen that the radius-reduced TurboSwirl configuration $(R$ $=60 \mathrm{~mm}$ ) can further lower the visible hump. Initially there is some splashing at approximately 3 seconds, but later the setup produces a lower visible hump with less fluctuation. This means less risk of mold flux entrapment and less risk of steel reoxidation. It is also seen that the visible hump decreases, as expected, as the filling proceeds. In addition, a properly tapered vertical runner can also create a similar effect, which also has been discussed by other researchers based on simulations of a reduced geometry model $[18,19]$.

It should also be mentioned that the mold powder release time and the release method should be adjusted to the current casting system. Therefore, the utilization of TurboSwirl can reduce the generation of macro nonmetallic inclusions, which mainly are formed due to reoxidation and mold flux entrapment.

3.2. Improved Steel Cleanliness in the Gating System. Although generations of researchers have tried to reduce the nonmetallic inclusion generation in the gating systems for years, it is still not possible to fully eliminate them. However, the presence of TurboSwirl creates new possibilities. Based on the results from the above section, a reduced model integrated with TurboSwirl was investigated using a Discrete Phase Model (DPM). A radius of $60 \mathrm{~mm}$ was also adopted for the component. After $4 \mathrm{~s}$ from the start of the filling process, alumina particles were injected from the inlet of the TurboSwirl for $0.1 \mathrm{~s}$. This corresponds to a total addition of $0.1 \mathrm{~g}$. The total simulation time for the filling process was 
TABLE 1: Particle distribution around the surface in the mold at $8 \mathrm{~s}$ from start of filling.

\begin{tabular}{lccccc}
\hline Diameter $(\mu \mathrm{m})$ & 2 & 10 & 30 & 100 & 200 \\
\hline $\begin{array}{l}\text { Distribution radius from } \\
\text { mold inlet axis }(\mathrm{dm})\end{array}$ & 1.91 & 1.90 & 2.56 & 1.89 & 1.98 \\
\hline $\begin{array}{l}\text { Distribution depth from the } \\
\text { surface in the mold }(\mathrm{dm})\end{array}$ & 1.00 & 0.94 & 1.01 & 0.80 & 0.35 \\
\hline Particle spread $\left(\mathrm{dm}^{3}\right)$ & 11.5 & 10.7 & 20.8 & 9.0 & 4.3 \\
\hline
\end{tabular}

TABLE 2: Gravitational terminal velocity for particles of different sizes.

\begin{tabular}{lccccc}
\hline $\begin{array}{l}\text { Diameter } \\
(\mu \mathrm{m})\end{array}$ & 2 & 10 & 30 & 100 & 200 \\
\hline $\begin{array}{l}\text { Terminal } \\
\text { velocity } \\
\left(\mathrm{m} \cdot \mathrm{s}^{-1}\right)\end{array}$ & $1.07 \cdot 10^{-6}$ & $2.68 \cdot 10^{-5}$ & $2.41 \cdot 10^{-4}$ & $2.68 \cdot 10^{-3}$ & $1.07 \cdot 10^{-2}$ \\
\hline
\end{tabular}

TABle 3: Maximum modified terminal velocity for particles of different sizes due to centripetal acceleration.

\begin{tabular}{lccccc}
\hline $\begin{array}{l}\text { Diameter } \\
(\mu \mathrm{m})\end{array}$ & 2 & 10 & 30 & 100 & 200 \\
\hline $\begin{array}{l}\text { Terminal } \\
\text { velocity } \\
\left(\mathrm{m} \cdot \mathrm{s}^{-1}\right)\end{array}$ & $6.48 \cdot 10^{-6}$ & $1.62 \cdot 10^{-4}$ & $1.46 \cdot 10^{-3}$ & $1.62 \cdot 10^{-2}$ & $6.48 \cdot 10^{-2}$ \\
\hline
\end{tabular}

$8 \mathrm{~s}$. Models used in the DPM description include pressure gradient force and virtual mass force. No consideration was taken with regard to turbulence effects on the particle motion since the near wall behavior was not of interest and also since an isotropic RANS turbulence model was used.

After $8 \mathrm{~s}$ from the filling start, the distribution of particles with different sizes is shown in Table 1. It can be found that the distribution of particles measured from the mold inlet axis generally has a diameter of $38-40 \mathrm{~cm}$. This is nearly equal to half of the mold diameter, which is $84.6 \mathrm{~cm}$. However, it is also shown that the particles having a $30 \mu \mathrm{m}$ diameter are distributed with a diameter of $51.2 \mathrm{~cm}$, which is a larger value than for the other diameters. From the particle distribution below the surface, it can be seen that the particles with a diameter of $200 \mu \mathrm{m}$ have the smallest range. In the present work, the size of particles is larger than $1 \mu \mathrm{m}$; therefore, the process can be studied with Newtonian mechanics. Here, the terminal velocity $\left(v_{t}\right)$ in Stokes' law is only used for a comparison, even though it is normally applied for a laminar flow. By considering the terminal velocity, which is shown in Table 2, it can be found that the particles having a diameter of $200 \mu \mathrm{m}$ have a highest velocity value of $1.1 \mathrm{~cm} \cdot \mathrm{s}^{-1}$. This is also seen in Table 1 where the large particles have a much smaller distribution range in the vertical direction (they cluster close to the surface). Thus, it promotes the removal rate of large nonmetallic inclusions to the mold flux during the uphill teeming process.

As has been discussed in the previous research by the authors, the TurboSwirl component can serve as a "collector," which prolongs the residence time of inclusions and promotes the agglomeration of inclusions before they reach the mold [11]. The residence time in the TurboSwirl setup (TS) was compared to a standard setup (SS). In this comparison the mold was omitted and the region surrounding the component was included, as can be seen in Figure 5. A relatively large number of particles (1500+) were injected in both systems during one time-step and later on tracked as they escaped from the top of the system. In both cases a steadystate flow field was calculated prior to the particle simulation. The particle residence time in both systems was measured by following the volume-average particle concentration in the solution domain. The results can be seen in Figures 6 and 7 . It is clear that the residence time in the TurboSwirl setup (SS) is much higher than the residence time in the standard setup (SS). Looking at the maximum residence time (i.e., when the concentration reaches zero), the increased residence time in the TS is a factor of four longer than in the SS case. From Figure 6 it is also clear that the particle size has a very small impact on the residence time. However, looking at Figure 7 it is seen that the particle residence time for 2,10 , and $30 \mu \mathrm{m}$ particles is very similar and that 100 and $200 \mu \mathrm{m}$ particles stand out as different. The $100 \mu \mathrm{m}$ particle concentration in the TS case decreases slightly faster compared to the smaller particles. However, there is almost no difference in the maximum residence time. For the $200 \mu \mathrm{m}$ particles in the TS case a much longer particle residence time can be seen. The maximum residence time (not seen in the figure) is 11.2 seconds, two to three times as high as the other particle sizes in the TS case and about 11 times as high as the SS case. The main difference in the results is attributed to the pressure gradient that builds up in the radial direction in the vertical pipe in the TurboSwirl. The rotational flow induces a virtual buoyancy on the particles in the direction of the axis due to this pressure gradient. The centripetal acceleration of the fluid gives rise to a modified terminal velocity in the direction of the axis. The profile of the centripetal acceleration for the present model is shown in Figure 8. It can be seen that the centripetal acceleration can reach a value of $59.3 \mathrm{~m} \cdot \mathrm{s}^{-2}$, which is more than five times higher than the gravitational acceleration. Therefore, the centripetal acceleration can have an influence in gathering nonmetallic inclusions towards the rotational center of a swirling flow. The comparison of the maximum modified terminal velocity using a TurboSwirl can be seen in Table 3. Although the modified terminal velocity in the radial direction can be increased by six times compared to the terminal velocity in the axial direction, the tendency for particles of a $2 \mu \mathrm{m}$ diameter to gather towards the center and leave for the mold is still very weak. It is also understood that the rotational flow decreases with an increasing distance from the TurboSwirl due to wall friction.

The nonmetallic inclusions can also be removed due to turbulent collisions. As mentioned above, a well-designed gating system can promote turbulent collisions by properly increasing the turbulence dissipation rate and the number concentration of inclusions in a relatively small volume. Nowadays, it is a trend that some steel companies try 


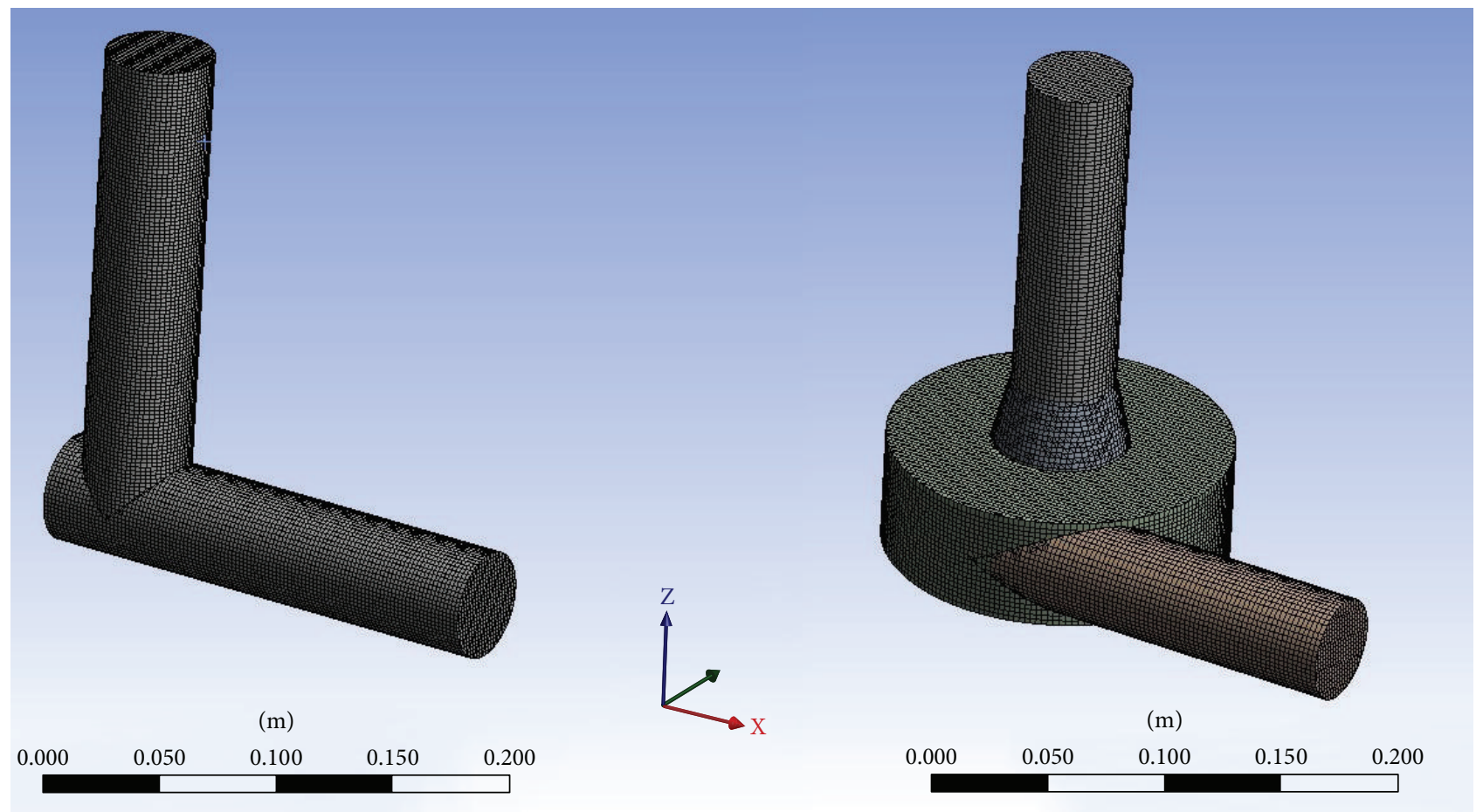

FIGURE 5: Investigation of the particle residence time in the component prior to the mold. Standard setup (SS) mesh to the left and TurboSwirl setup (TS) mesh to the right.

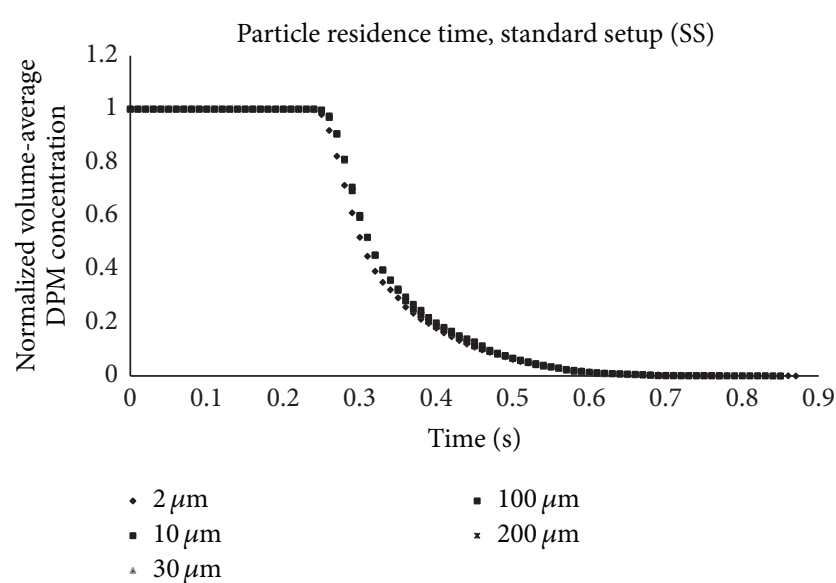

Figure 6: Particle residence in a standard component just before the mold, for alumina particles with a size range of $2-200 \mu \mathrm{m}$.

to reduce the size of inclusions to an even smaller scale, that is, a submicron size. Meanwhile, it is also necessary to remove nonmetallic inclusions of a larger size, that is, in the order of millimeter. The $200 \mu \mathrm{m}$ particles in the present case simulations are close to the average Kolmogorov length scale and because of this the validity of (2) could be questioned; however recent experimental studies have shown that the acceleration and velocities of inertial particles with sizes larger than the Kolmogorov length scale in an intense turbulent flow can still be characterized by introducing a correction based on the particle Reynolds number [20]. Since

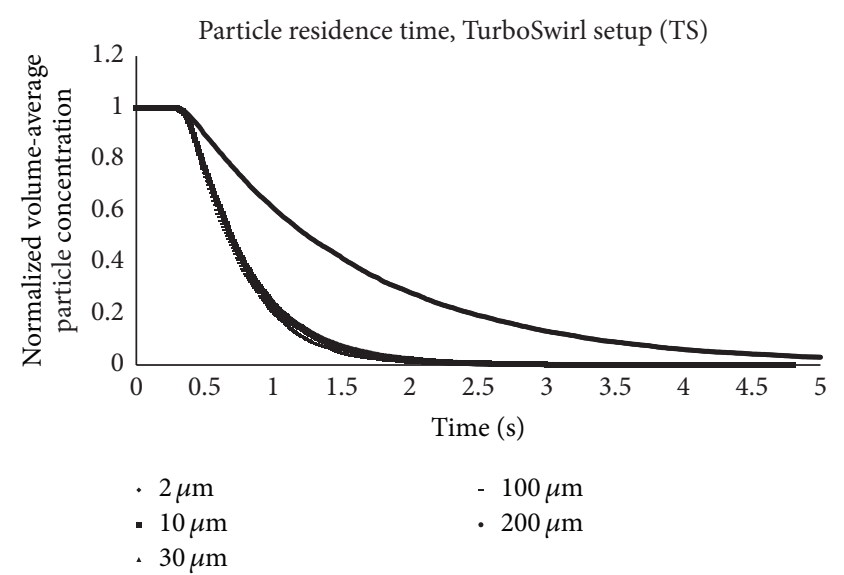

Figure 7: Particle residence in a TurboSwirl component just before the mold, for alumina particles with a size range of $2-200 \mu \mathrm{m}$.

no agglomeration of particles was considered in the present case, inclusions with a diameter of $2 \mu \mathrm{m}$ and $200 \mu \mathrm{m}$ were investigated for both a setup with swirl (TS) and a setup with no swirl (L).

The average dissipation rate in the gating system, excluding the mold, during the filling process is shown in Figure 9. It can be seen that the average dissipation rate in a swirl setup is about $40 \%$ higher than that in a setup with no swirl, which can promote the removal of nonmetallic inclusions due to turbulent collisions. In addition, it can also be noticed that the dissipation rate decreases as the filling proceeds. 


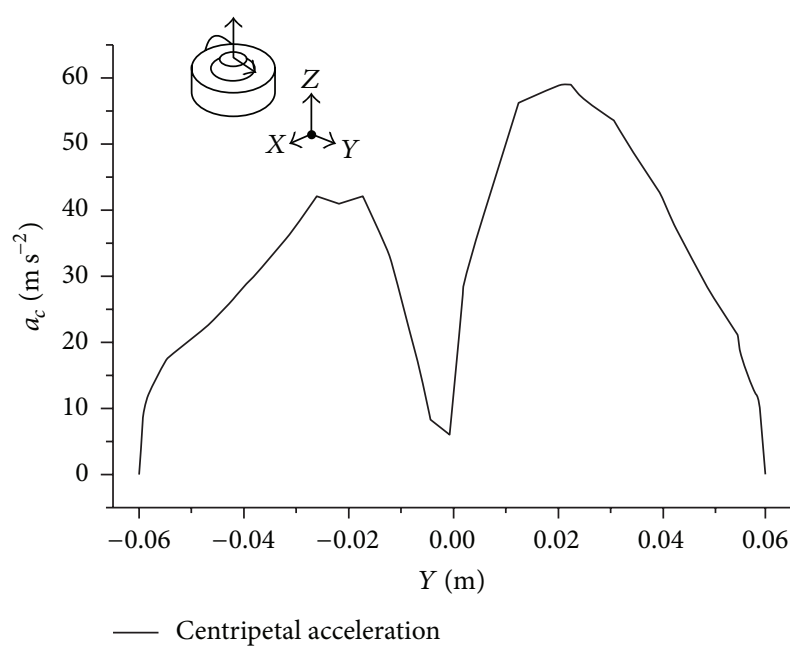

FIGURE 8: Centripetal acceleration profile in the TurboSwirl along a line in the $x y$-plane, where the axis of rotation is the $z$-axis and the $x y$-plane is located at the entrance to the converging part of the TurboSwirl.

TABLE 4: Comparison of the range of dissipation rates for a $2 \mu \mathrm{m}$ inclusion size.

\begin{tabular}{lcc}
\hline Model & TS (swirl) & L (no swirl) \\
\hline Minimum dissipation rate $\left(\mathrm{m}^{2} \cdot \mathrm{s}^{-3}\right)$ & 0.00122 & 0.000809 \\
Maximum dissipation rate $\left(\mathrm{m}^{2} \cdot \mathrm{s}^{-3}\right)$ & 14.26512 & 25.598 \\
\hline
\end{tabular}

This indicates that the removal efficiency is reduced when a ferrostatic pressure builds up in mold. A comparison of the range of dissipation rate of the entire system is shown in Table 4. It can be seen that the range of dissipation rate for the no swirl setup is much larger than that for the swirl setup. This means that a much calmer filling condition is achieved in the swirl setup compared to the setup with no swirl.

The number concentration of nonmetallic inclusions is another important factor which determines the collision rate of inclusions due to turbulent collisions. In the swirl setup, it can be divided into three parts, that is, a generation part (component), a developing part (vertical runner), and a result part (mold). The developing part is of great interest to study as it is an essential region to control the distribution of inclusions as is shown in Figure 10. Owing to the nature of a swirling flow, the profile of the inclusion population in a vertical runner is different from that in a no swirl setup, as can be seen in Figure 11. Here, the percentage represents the inclusion population in the vertical runner out of the total inclusion population injected into the model. It can be seen that the inclusion population in the vertical runner of a no swirl setup decreases continuously whereas a monomodal result appears in the vertical runner of a swirl setup due to a swirling flow. In the vertical runner of a no swirl setup, a higher value presents for particle with a diameter of $200 \mu \mathrm{m}$. This results from a recirculation region in the elbow of the runners, which is due to an uneven flow distribution. In the swirl setup, the component prolongs the residence time for inclusions in the TurboSwirl. This leads to a more even

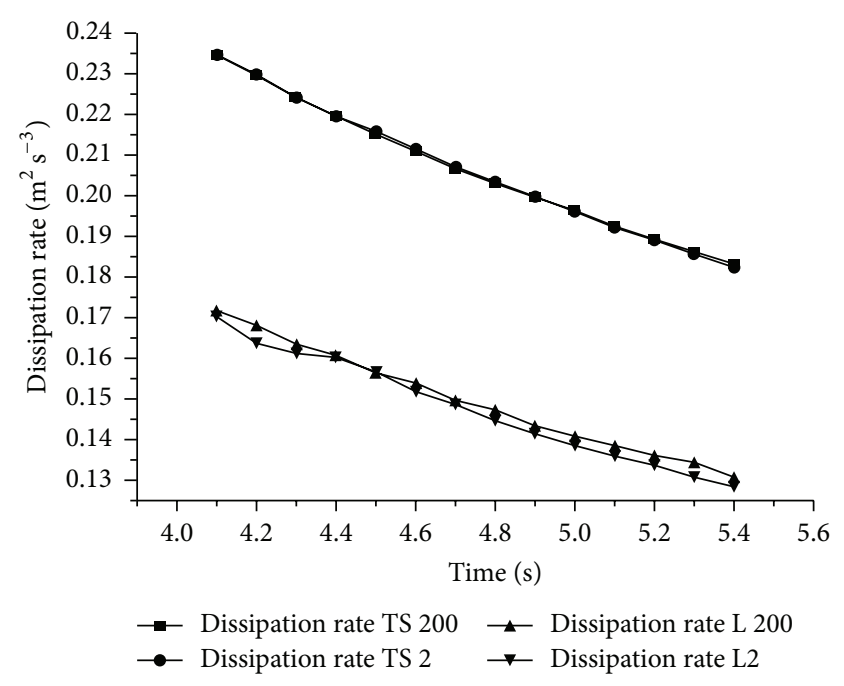

FIGURE 9: Average dissipation rate in the swirl setup (TS) and no swirl setup (L) for 2 and $200 \mu \mathrm{m}$ inclusion sizes.

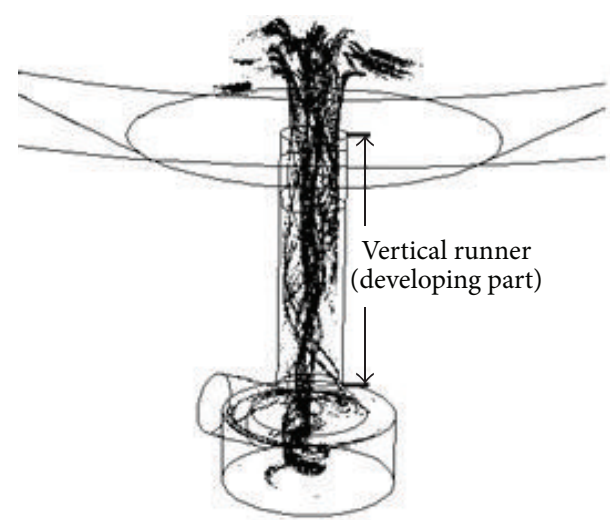

Figure 10: Inclusion distribution in the TurboSwirl, during filling.

profile for inclusions entering the vertical runner. In addition, the centripetal acceleration promotes nonmetallic inclusions gathering towards the rotational center, which results in a nonlinear increase of the profile. Note that the inclusion injection is only for 0.1 seconds; a continuous injection would create a different pattern. The current information serves to illustrate that the inclusion residence time in the vertical runner is very much affected by the usage of swirl.

Due to the nature of a swirling flow, it causes the lighter nonmetallic inclusions to have a centering movement. As an evaluation method a cylindrical volume was investigated for inclusions in the vertical runner. The cylinder diameter was analyzed by 1,2 , and $3 \mathrm{~cm}$ in a concentric way (the diameter of the vertical runner is $4.5 \mathrm{~cm}$ ). The profile of the inclusion population evolution in the vertical runner is studied. The results for $200 \mu \mathrm{m}$ and $2 \mu \mathrm{m}$ are shown in Figures 12 and 13 , respectively. It can be seen that a maximum value of $48 \%$ out of the total inclusion population of a $200 \mu \mathrm{m}$ size in the vertical runner can be concentrated in the volume that has a diameter of $1 \mathrm{~cm}$. The corresponding value is $51 \%$ for inclusions of a $2 \mu \mathrm{m}$ diameter. Here, the maximum 


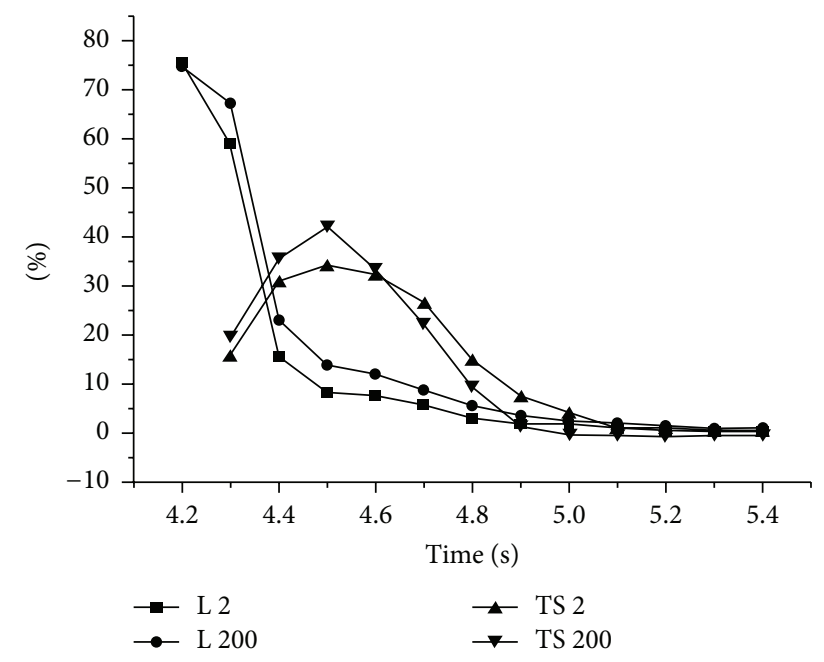

FIGURE 11: Inclusion population in a swirl setup (TS) and in a no swirl setup (L) for 2 and $200 \mu \mathrm{m}$ inclusion sizes. The percentage is given from number of inclusions to the total number of injected inclusions, present in the TurboSwirl.

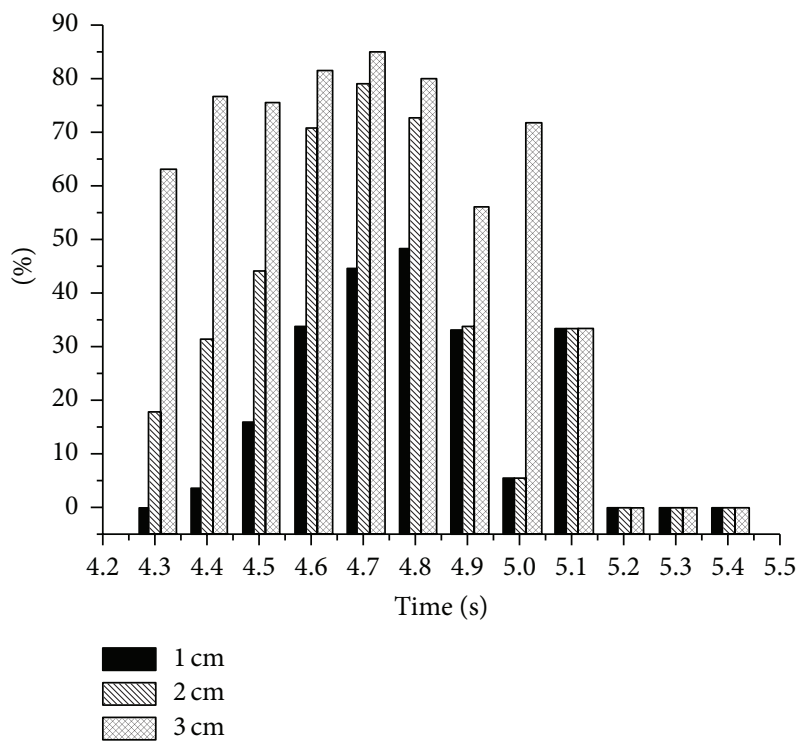

FIGURE 12: Inclusion population evolution for inclusions of a $200 \mu \mathrm{m}$ size in the vertical runner. The three cases show inclusions in an imaginary cylinder centered at the axis of the runner, with a diameter of 1,2 , and $3 \mathrm{~cm}$, respectively. The percentage is given from number of inclusions to the total number of injected inclusions, present in the corresponding volume.

efficiency takes place. In addition, inclusions of a $200 \mu \mathrm{m}$ size can be concentrated early. However, only $16 \%$ and $1.1 \%$ of the total inclusion population of sizes $200 \mu \mathrm{m}$ and $2 \mu \mathrm{m}$, respectively, are concentrated in the analyzed volume center of $1 \mathrm{~cm}$ diameter when the peak value of inclusion population occurs in the vertical runner. The peak number concentration of inclusions within a diameter of $1 \mathrm{~cm}$ occurs at $4.6 \mathrm{~s}$ for inclusions of a $200 \mu \mathrm{m}$ diameter with a $33.7 \%$ value and at $4.8 \mathrm{~s}$ for $2 \mu \mathrm{m}$ particles with a $34.8 \%$ value. Hence, a further

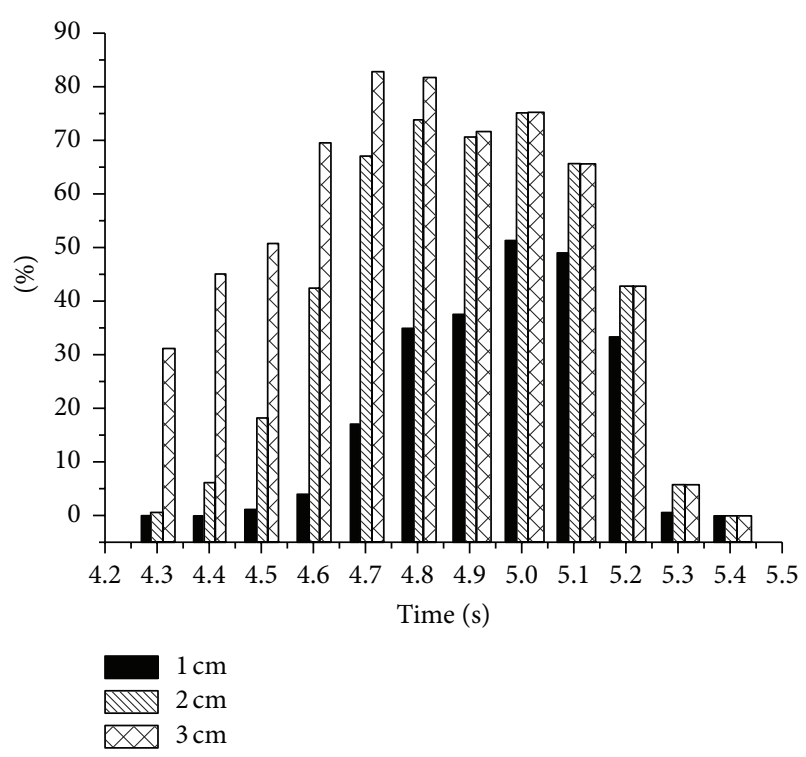

FIGURE 13: Inclusion population evolution for inclusions of a $2 \mu \mathrm{m}$ size in the vertical runner. The three cases show inclusions in an imaginary cylinder centered at the axis of the runner, with a diameter of 1,2 , and $3 \mathrm{~cm}$, respectively. The percentage is given from number of inclusions to the total number of injected inclusions, present in the corresponding volume.

optimization of nonmetallic inclusions concentration in the center is suggested in future work.

The profile of the number concentration of nonmetallic inclusions located within a diameter of $1 \mathrm{~cm}$ of the vertical runner axis is shown in Figure 14. It can be seen that the number concentration for the no swirl setup decreases very fast due to a lack of a centripetal acceleration. However, in the vertical runner, the number concentration for the swirl setup can reach a value of up to $11.4 \%$ for inclusions of a $200 \mu \mathrm{m}$ size and $5.1 \%$ for inclusions of a $2 \mu \mathrm{m}$ diameter out of the total nonmetallic inclusions presented in the gating system. It can clearly be seen that the utilization of TurboSwirl can increase the number concentration of nonmetallic inclusions in the gating system, especially for inclusions of large sizes. Therefore, the collision rate of inclusions due to turbulent collisions can also be enhanced.

\section{Conclusions}

The present study focused on revealing the effects TurboSwirl has on improving the steel cleanliness. It is found that the utilization of TurboSwirl can improve the steel cleanliness by reducing mold flux entrapment and by increasing the nonmetallic inclusions collision rate due to both Stokes collisions and turbulent collisions. The following specific conclusions may be summarized from the current study:

(1) The possibility of mold flux entrapment can further be reduced by utilization of swirl technology to decrease the visible hump formed during casting, that is, by minimizing the open eye on the rising steel surface. By properly reducing the radius of TurboSwirl and 


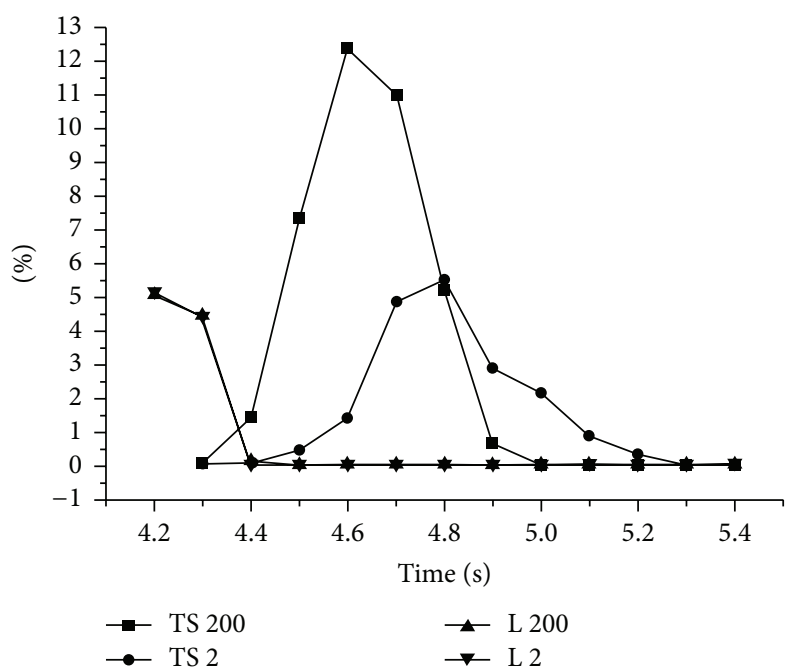

FIGURE 14: Number concentration of nonmetallic inclusions located within an imaginary cylinder centered at the vertical runner axis, with a diameter of $1 \mathrm{~cm}$. TurboSwirl setup (TS) and no swirl setup (L) with two different inclusion diameters of 2 and $200 \mu \mathrm{m}$. The percentage is given from number of inclusions to the total number of injected inclusions, present in the corresponding volume.

adopting a tapered mold entrance nozzle with an adequate developing region for the steel flow in a swirl setup, the swirl can be tailored to most uphill teeming systems. The steel cleanliness can further be improved in the mold when the mold powder release time and the release method are adjusted to the current casting setup.

(2) The removal rate of large nonmetallic inclusions by mold flux in the uphill teeming process can be promoted by using TurboSwirl. For inclusions of small sizes, the component can serve as a "collector," which prolongs the residence time of inclusions and promotes inclusion separation from the steel. The residence time for inclusions in the TurboSwirl component is approximately four times longer compared to a conventional setup at the same flow rates.

(3) The utilization of TurboSwirl can promote turbulent collisions by properly increasing the turbulence dissipation rate and the number concentration of inclusions in a relatively small volume. The average dissipation rate in the swirl setup is about $40 \%$ higher than that in a setup without swirl. The maximum efficiency for gathering inclusions towards the center volume, within a diameter of $1 \mathrm{~cm}$ of the axis, can be up to $48 \%$ out of the total inclusion population in the vertical runner for inclusions in size of $200 \mu \mathrm{m}$ and $51 \%$ for that of inclusions with a diameter of $2 \mu \mathrm{m}$. The number concentration for the swirl setup can reach up to $11.4 \%$ for inclusions of a $200 \mu \mathrm{m}$ size and $5.1 \%$ for inclusion with a diameter of $2 \mu \mathrm{m}$. This compared to the total nonmetallic inclusions present in the gating system.
In summary, the use of TurboSwirl reveals a great potential for improving the steel cleanliness. The inclusions distribution and collision rate are related to the Kolmogorov scale in the filling process. The boundary condition, that is, teeming rate, has great influence in removing nonmetallic inclusions in uphill teeming process. Therefore, it is highly desirable to perform plant trial or physical modeling verification for the model in the future.

\section{Nomenclature}

$\eta$ : Kolmogorov length scale $[\mathrm{m}]$

$v$ : Kinematic viscosity $\left[\mathrm{m}^{2} \mathrm{~s}^{-1}\right]$

$\varepsilon:$ Dissipation rate of turbulence kinetic energy $\left[\mathrm{m}^{2} \mathrm{~s}^{-3}\right]$

$k$ : Turbulence kinetic energy $\left[\mathrm{m}^{2} \mathrm{~s}^{-2}\right]$

$W_{t}$ : Turbulence volume collision rate $\left[\mathrm{m}^{3} \mathrm{~s}^{-1}\right]$

$W_{s}$ : Stokes collision volume $\left[\mathrm{m}^{3} \mathrm{~s}^{-1}\right]$

$W$ : Total volume collision rate $\left[\mathrm{m}^{3} \mathrm{~s}^{-1}\right]$

$n_{i}$ : Number concentration of particle $i\left[\mathrm{~m}^{-3}\right]$

$v_{t}$ : Terminal velocity $\left[\mathrm{m} \mathrm{s}^{-1}\right]$

$r$ : Particle radius $[\mathrm{m}]$

$R$ : TurboSwirl radius [m]

$\rho_{p}$ : Density of particle (inclusion) $\left[\mathrm{kg} \mathrm{m}^{-3}\right]$

$\rho_{f}$ : Density of liquid (steel) $\left[\mathrm{kg} \mathrm{m}^{-3}\right]$

$g$ : Gravitational acceleration $\left[\mathrm{m} \mathrm{s}^{-2}\right]$

$\mu$ : Viscosity $[\mathrm{Pas}]$

$a_{c}:$ Centripetal acceleration $\left[\mathrm{m} \mathrm{s}^{-2}\right]$

$v_{t}^{\prime}$ : Modified terminal velocity $\left[\mathrm{m} \mathrm{s}^{-1}\right]$

$\dot{\gamma}$ : $\quad$ Fluid deformation rate $\left[\mathrm{s}^{-1}\right]$

A: Hamaker constant [J].

\section{Conflict of Interests}

The authors declare that there is no conflict of interests regarding the publication of this paper.

\section{Acknowledgments}

The authors wish to give their deepest gratitude to Jernkontoret (Swedish Steel Producers Association) and Vinnova for financial support to this study. Furthermore, they are thankful for the input from the members of the committee JK24053.

\section{References}

[1] P. Sjödin, P. Jönsson, M. Andreasson, and A. Winquist, Scandinavian Journal of Metallurgy, vol. 26, p. 41, 1997.

[2] L. Zhang and B. G. Thomas, "State of the art in the control of inclusions during steel ingot casting," Metallurgical and Materials Transactions B, vol. 37, no. 5, pp. 733-761, 2006.

[3] Z. Tan, M. Ersson, and P. G. Jönsson, "Mathematical modeling of initial filling moment of uphill teeming process considering a trumpet," ISIJ International, vol. 51, no. 9, pp. 1461-1467, 2011.

[4] D.-Y. Sheng, M. Söder, P. Jönsson, and L. Jonsson, "Modeling micro-inclusion growth and separation in gas-stirred ladles," Scandinavian Journal of Metallurgy, vol. 31, no. 2, pp. 134-147, 2002. 
[5] Y. Miki, B. G. Thomas, A. Denissov, and Y. Shimada, "Model of inclusion removal during RH degassing of steel," Iron and Steelmaker, vol. 24, no. 8, pp. 31-38, 1997.

[6] P. G. Saffman and J. S. Turner, "On the collision of drops in turbulent clouds," Journal of Fluid Mechanics, vol. 1, no. 1, pp. 16-30, 1956.

[7] L. Ragnarsson and D. Sichen, "Erosion of runners in ingot casting," in Proceedings of the AISTech, p. 1077, AIST, Warrendale, Pa, USA, 2009.

[8] V. Singh, P. A. L. Atanu Ranjan, and P. Panigrahi, "Numerical simulation of flow-induced wall shear stress to study a curved shape billet caster tundish design," ISIJ International, vol. 48, no. 4, pp. 430-437, 2008.

[9] K. Marx, S. Rödl, S. Schramhauser, and M. Seemann, "Optimization of the filling and solidification of large ingots," $L a$ Metallurgia Italiana, no. 11-12, 2014.

[10] German Patent no. DE285999, Jörpeland, 1914.

[11] Z. Tan, M. Ersson, and P. G. Jönsson, "Modeling of initial mold filling with utilization of swirl blades," ISIJ International, vol. 52, no. 6, pp. 1066-1071, 2012.

[12] Y. Yang, P. G. Jönsson, Z. Su, J. He, and K. Nakajima, “Design of magnetic fields for half and full type electromagnetic swirl flow generators," Steel Research International, vol. 86, no. 4, pp. 361-374, 2015.

[13] Y. Yang, P. G. Jönsson, M. Ersson, and K. Nakajima, "Inclusion behavior under a Swirl flow in a submerged entry nozzle and mold," Steel Research International, vol. 86, no. 4, pp. 341-360, 2015.

[14] Z. Tan, M. Ersson, P. Lidegran, and P. G. Jönsson, "Uphill teeming utilizing TurboSwirl to control flow pattern in mold," Steel Research International, vol. 84, no. 9, pp. 837-844, 2013.

[15] K. Higashitani, K. Yamaguchi, Y. Matsuno, and G. Hosokawa, "Turbulent coagulation of particles dispersed in a viscous fluid," Journal of Chemical Engineering of Japan, vol. 16, no. 4, pp. 299304, 1983.

[16] H. Lamb, Hydrodynamics, Cambridge University Press, Cambridge, UK, 1932.

[17] ANSYS User's Guide Version 15.0, http://www.ansys.com.

[18] R. Eriksson, L. Jonsson, and P. G. Jönsson, "Effect of entrance nozzle design on the fluid flow in an ingot mold during filling," ISIJ International, vol. 44, no. 8, pp. 1358-1365, 2004.

[19] L. Hallgren, S. Takagi, A. Tilliander, S. Yokoya, and P. Jönsson, "Effect of nozzle type and swirl on flow pattern for initial filling conditions in the mould for up-hill teeming," Steel Research International, vol. 78, no. 3, pp. 254-259, 2007.

[20] H. Xu and E. Bodenschatz, "Motion of inertial particles with size larger than Kolmogorov scale in turbulent flows," Physica D: Nonlinear Phenomena, vol. 237, no. 14-17, pp. 2095-2100, 2008. 


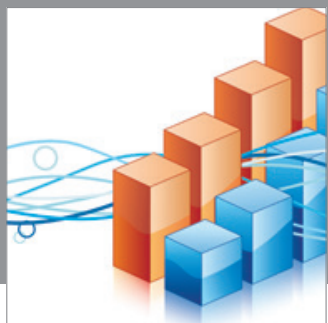

Advances in

Operations Research

mansans

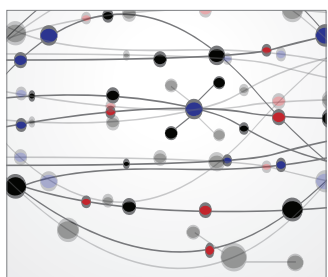

The Scientific World Journal
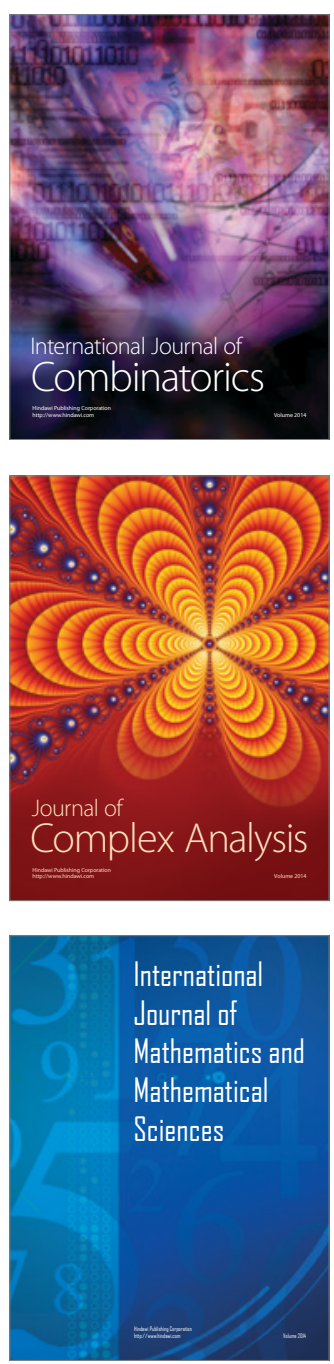
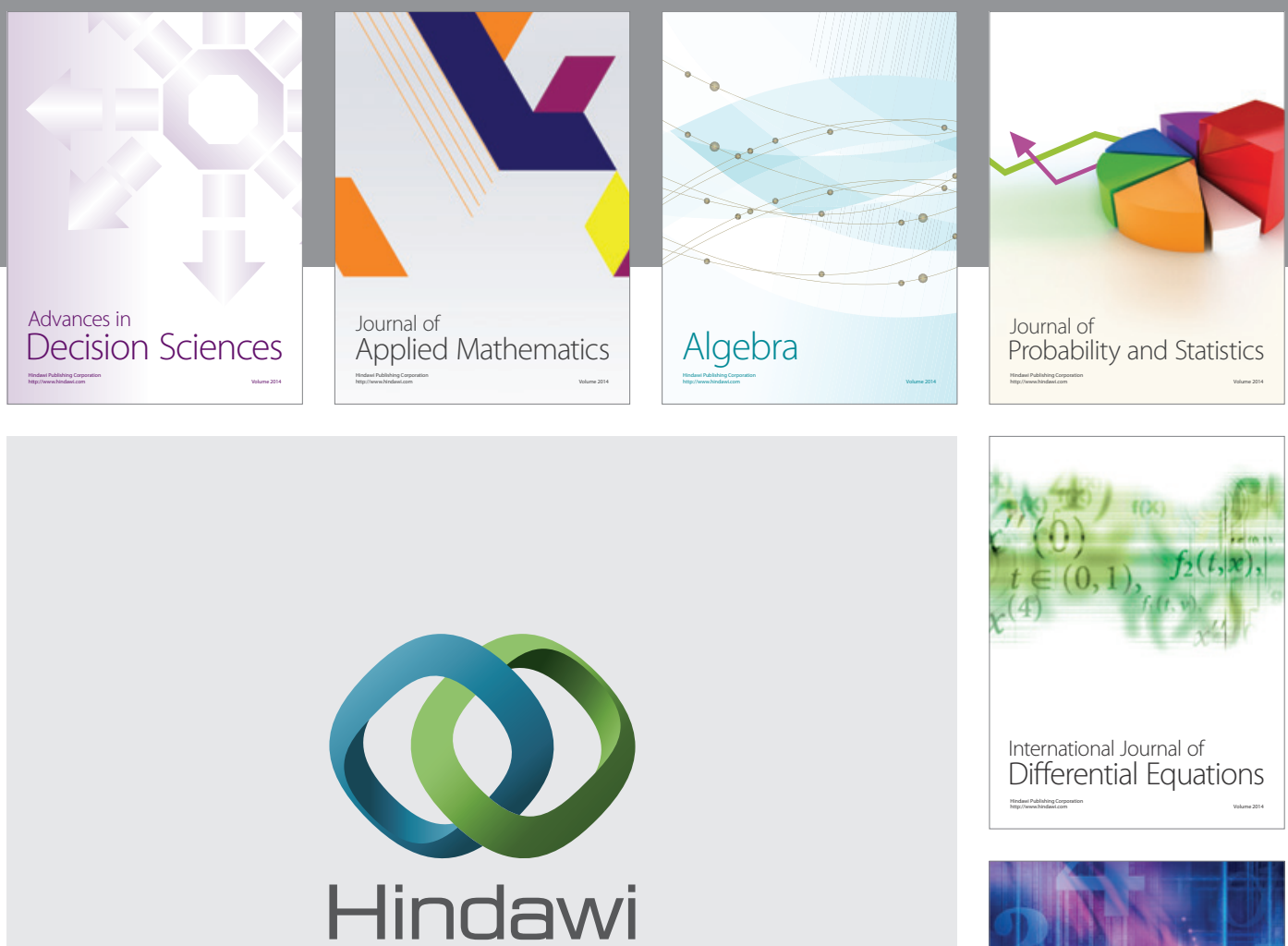

Submit your manuscripts at http://www.hindawi.com
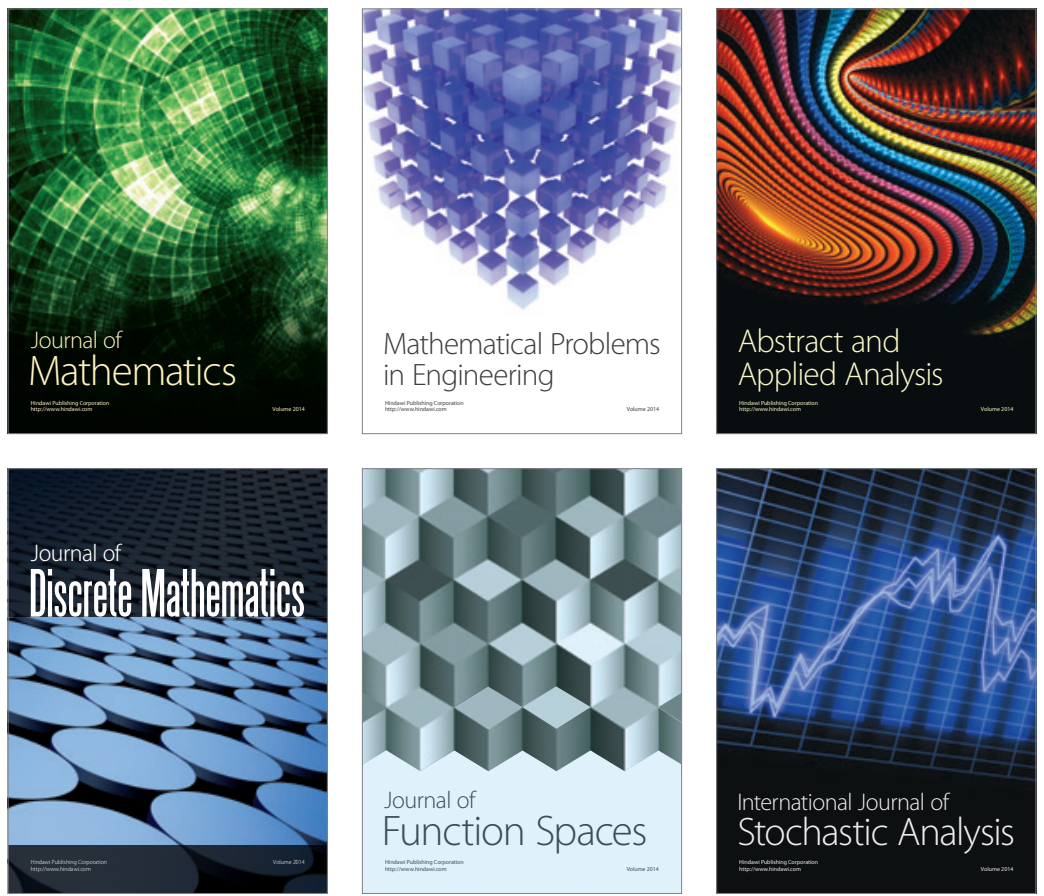

Journal of

Function Spaces

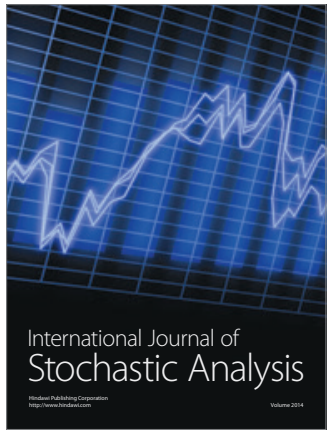

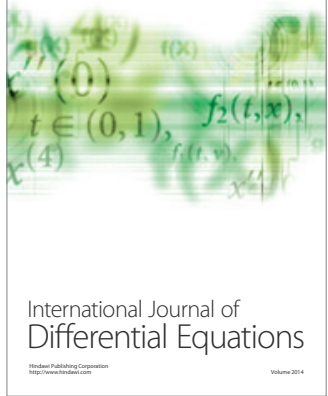
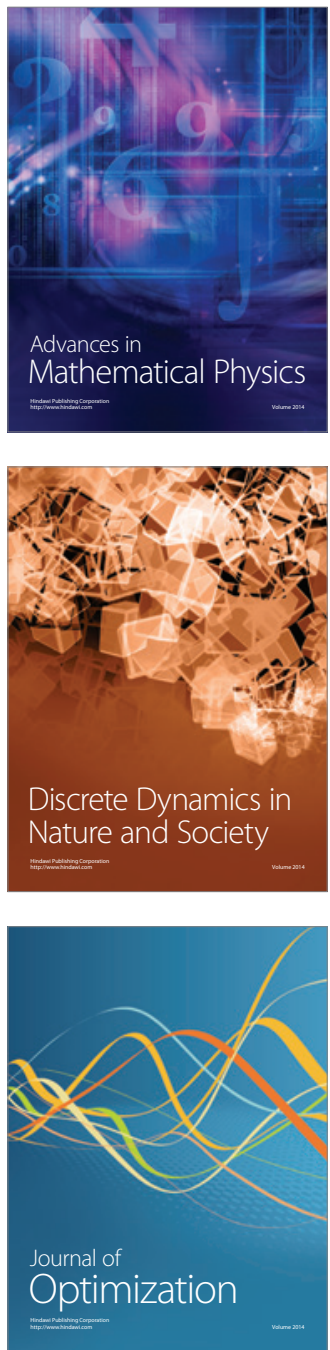